Journal of Engineering and Applied Sciences 14 (15): 5152-5156, 2019

ISSN: 1816-949X

(C) Medwell Journals, 2019

\title{
A Novel Method for Calculating a Fingerprint Gradient
}

\author{
${ }^{1,2}$ H.I. Wahhab, ${ }^{1,3}$ A.N. Alanssari, ${ }^{1}$ V.J. Gudkov and ${ }^{1}$ O.V. Behtold \\ ${ }^{1}$ South Ural State University, Chelyabinsk, Russian Federation \\ ${ }^{2}$ University of Kerbala, Kerbala, Republic of Iraq \\ ${ }^{3}$ University of Kufa, Najaf, Republic of Iraq \\ haider.wahhab@uokerbala.edu.iq, alaan.azeez@uokufa.edu.iq,diana@sonda.ru, \\ olegbehtold@gmail.com
}

\begin{abstract}
Fingerprint identification systems are one of the most well-known and widely-used biometrics because of their inherent ease in acquisition, the numerous sources (ten fingers) available for collection and their established use by law enforcement and immigration services. These systems rely on the unique biological characteristics of individuals to accurately verify their identities. To get reliable and accurate verification results, these systems need high-quality images. Fingerprint image quality is achieved by using noise-free images in the pre-processing and filtering stages. In this study, we utilized several techniques to improve the image such as smoothing, up sampling and gradient. This combination was made by using two coefficient parameters $(\alpha, \beta)$ with different values to enhance the quality of images and remove the unwanted distortion or noise that affect a fingerprint recognition system. The method is applied in the pre-processing stage to get a noise-free fingerprint image with high accuracy factor.
\end{abstract}

Key words: Image processing, fingerprint recognition, smoothing, fingerprint orientation field, gradient, results

\section{INTRODUCTION}

Biometric identification systems are based on the ways in which individuals can be uniquely identified through one or more distinguishing biological traits such as fingerprints, hand geometry, earlobe geometry, retina and iris patterns, voice waves, Deoxyribonucleic Acid (DNA) and signatures (Imamverdiyev et al., 2013). Fingerprint identification system is one of the most popular biometric technologies and the performance of a fingerprint image matching algorithm depends heavily on the quality of the input fingerprint images (Kayaoglu et al., 2015).

Papillary lines allow building a flow map for a fingerprint. On the basis of the flow map, it is possible to recognize loops, deltas and whorls their position and orientation. The advantages of the fingerprint image recognition are the use of relatively simple, inexpensive scanners and the higher efficiency of the matching process in the case of low resolution images (Nagar et al., 2010). A good quality fingerprint image has about 40-100 minutiae. Minutiae are points of interest in a fingerprint tip such as ridge bifurcation and ending (Cappelli et al., 2010a, b). The bifurcation is a single ridge that divides into two ridges while the ending is a ridge that ends abruptly (Khfagy et al., 2016) as shown in Fig. 1.

Images are those types of data that are widely used in various areas (Xu and Veldhuis, 2010a-c). Noisy and poor captured images that result in false generation and

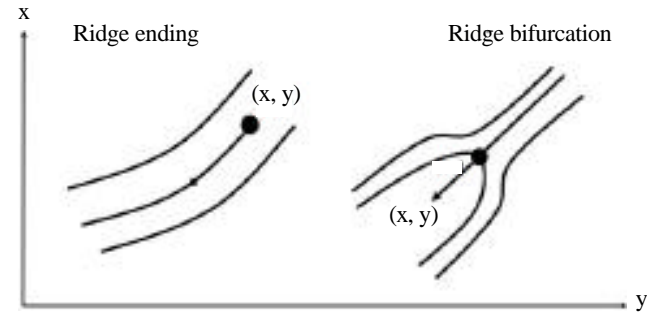

Fig. 1: Ridge bifurcation and ridge ending

loss genuine of minutiae are a challenge in any fingerprint image recognition system (Xu and Veldhuis, 2010a-c). Noise in fingerprint images may occur due to variations in skin and impression conditions such as scars, humidity, dirtand non-uniform contact with the fingerprint capture device which distorts the clarity of the ridge structures (Xu and Veldhuis, 2010a-c). The main goal includes building a trustworthy data model to represent randomly oriented lines of the fingertip and finding ways to compare the models with the highest accuracy in the minutiae matching stage (Cappelli et al., 2010). Any identification system design should consists of two major steps, namely, enrollment stage and verification stage (Hiew et al., 2010). The enrollment stage includes capturing a fingerprint image from a sensor device with the following extractor identifying the features of the fingerprint image and storing them into an enrolling template in system database. In the

Corresponding Author: H. I. Wahhab, University of Karbala, Karbala, Republic of Iraq haider.wahhab@uokerbala.edu.iq 
verification stage, matching algorithms are used to compare previously stored templates of fingerprints against the candidate's fingerprints for authentication purposes (Can and Lin, 2009).

\section{MATERIALS AND METHODS}

Fingerprint image capture: Get an accurate image of a fingerprint through a scanner with a resolution of $500 \mathrm{dpi}$. A resolution of 500 dpi is also the standard fingerprint resolution of the federal bureau of investigation for Automatic Fingerprint Identification Systems (AFIS) using minutiae (Jain et al., 2007). At this accuracy, the proximity of the ridges to each other leads to the possibility of some distortions in the image of the fingerprint as a gluing of adjacent ridges because of the fingerprint image taken not clear or because of a wound in the finger skin. Also, some dirt on the finger skin or on the scanner may significantly affect the image quality, so, we double the image to 1000 dpi using the following Eq. 1:

$$
\begin{aligned}
& \mathrm{a}(\mathrm{x}, \mathrm{y})=\left(\mathrm{b}(\mathrm{x}, \mathrm{y})^{*} \tau+\mathrm{b}(\mathrm{x}+1, \mathrm{y})(1-\tau)\right)^{*} \tau+ \\
& \left(\mathrm{b}(\mathrm{x}, \mathrm{y}+1)^{*} \tau+\mathrm{b}(\mathrm{x}+1, \mathrm{y}+1)(1-\tau)\right)^{*}(1-\tau)
\end{aligned}
$$

where, $\tau$ is equal to $1 / 2$ in case of doubling. Potential deformations can be reduced or minimized by increasing the image resolution as shown in Fig. 2 and 3.

Image smoothing: Smoothing filters are used for blurring and noise reduction. Blurring is employed in preprocessing steps such as removal of little details from a picture before (large) object extraction and bridging of little gaps in lines or curves.

Noise reduction will be accomplished by blurring with a linear filter and in addition by nonlinear filtering (Maponi et al., 2017; Gonzalez and Woods, 2002a, b).

In this study, we calculated a quarter of the output value of the filter, since, we also doubled the brightness of the image. Linear filtering of the image of 1000 dpi with a $5 \times 5$ filter mask is given by the expression (Gonzalez and Woods, 2002a, b):

$$
f(x, y)=\left(\sum_{i=-2}^{2} \sum_{j=2}^{2} i . j\right) \cdot f(x+i, y+j)
$$

We used the value (zero) in the center of the filter to get the best smoothing results shown in Fig. 4. After smoothing, we calculate average brightness for every $2 \times 2$ block and prepare the similar smoothed image for $500 \mathrm{dpi}$ as shown in Fig. 5:

$$
S^{\prime}=(f(x, y)+f(x+1, y)+f(x, y+1)+f(x+1, y+1) / 4
$$

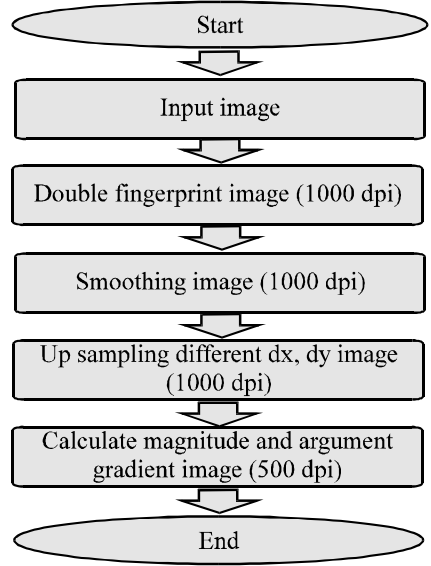

Fig. 2: Schematic diagram for fingerprint gradient measuring

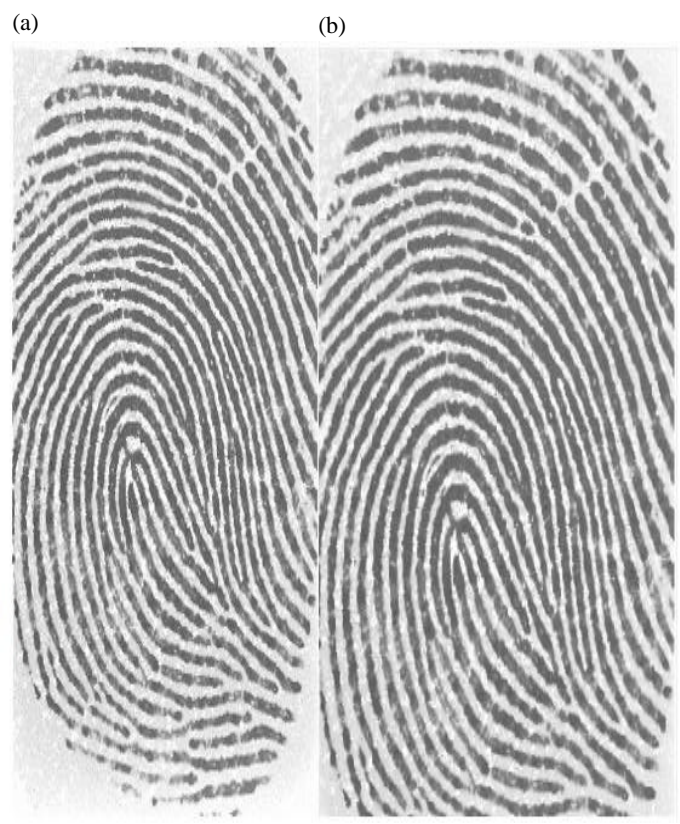

Fig. 3: Fingerprint image: a) 500 dpi and b) 1000 dpi

\begin{tabular}{|c|c|c|c|c|}
\hline 1 & 0 & 1 & 0 & 1 \\
\hline 0 & 0 & 0 & 0 & 0 \\
\hline 1 & 0 & 0 & 0 & 1 \\
\hline 0 & 0 & 0 & 0 & 0 \\
\hline 1 & 0 & 1 & 0 & 1 \\
\hline
\end{tabular}

Fig. 4: Smoothing filter $5 \times 5,1000 \mathrm{dpi}$ 


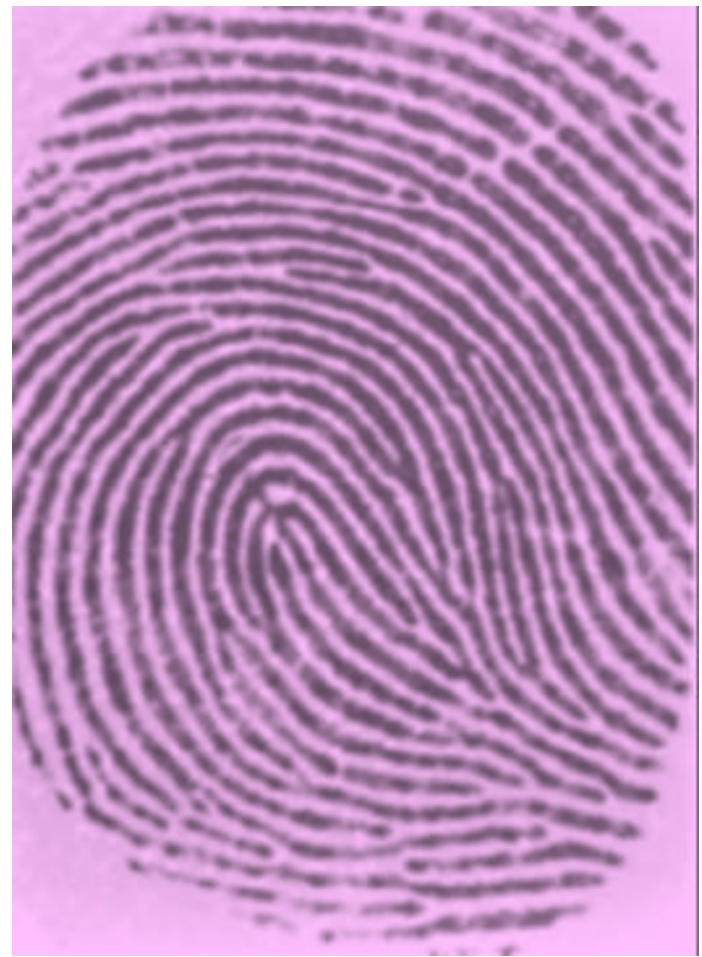

Fig. 5: Smoothing image $500 \mathrm{dpi}$

\section{RESULTS AND DISCUSSION}

Difference up sampling: In fingerprint recognition programs (AFIS), the Fingerprint Orientation Field (FOF) feature is a global characteristic. Traditional methods based on the FOF are commonly usedbut they are very sensitive to noise resulting from low quality fingerprints such as oil, moisture, dust, wrinkles, scars, breaks, etc., (Tashk et al., 2010). The way to improve the performance of fingerprint recognition is to establish a good model for the FOF (Bian et al., 2018) which is of great importance for image processing (Gu and Zhou, 2003). A well-known method to improve the FOF is the Convolutional Neural Network $(\mathrm{CNN})$. There are many non-linear functions, amongst which max pooling is the most common. It splits the input picture into a group of non-overlapping rectangles and outputs the maximum for each such sub-region. The pooling layer serves to piecemeal reduce the spatial size of the representation to minimize the number of parameters and amount of calculation in the network.

In this study, we suggest an effective algorithm to find the FOF (Ma et al., 2010). We use smoothed image (1000 dpi) and calculate up sampling differences by specifying a block of $2 \times 2$. We accumulate the sum of the neighbors into block and place the result in special variables for each block. The sum of the block $h$ (the yellow color) is accumulated and stored in the variable

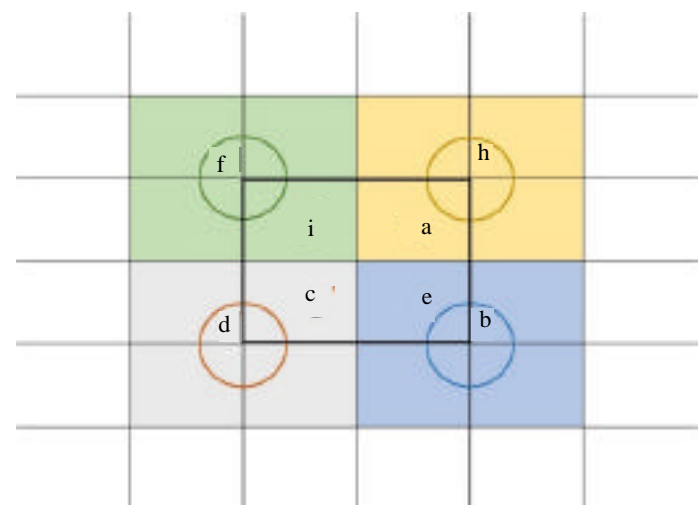

Fig. 6: Up sampling

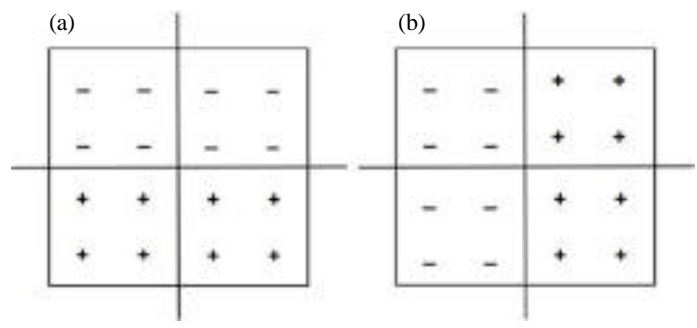

Fig. 7: The scheme to calculate; a) The difference along $\mathrm{y}$ axis and $\mathrm{b}$ ) The difference along $\mathrm{x}$ axis

$\mathrm{Sh}$; the sum of block $\mathrm{b}$ (the blue color) in variable $\mathrm{Sb}$; the sum of block d (the gray color) in the variable Sd and the sum of block $\mathrm{f}$ (the green color) in the variable $\mathrm{Sf}$ (Doroz et al., 2018; Lee et al., 2008) as shown in Fig. 6. Each block is accumulated in the form of:

$$
\begin{aligned}
& \operatorname{Sh}(x, y)=f(x+1, y-1)+f(x+2, y-1)+ \\
& f(x+1, y)+f(x+2, y) \\
& \operatorname{Sb}(x, y)=f(x+1, y+1)+f(x+2, y+1)+ \\
& f(x+1, y+2)+f(x+2, y+2) \\
& \operatorname{Sd}(x, y)=f(x-1, y+1)+f(x, y+1)+ \\
& f(x-1, y+2)+f(x, y+2) \\
& \operatorname{Sf}(x, y)=f(x-1, y-1)+f(x, y-1)+ \\
& f(x-1, y)+f(x, y)
\end{aligned}
$$

The difference along $\mathrm{x}$-axis based on the image 1000 dpi is calculated in the form:

$$
\mathrm{dx}(\mathrm{x}, \mathrm{y})=\mathrm{Sh}+\mathrm{Sb}-\mathrm{Sf}-\mathrm{Sd}
$$

and the similar difference along y axis (Fig. 7 and 8): 
(a)

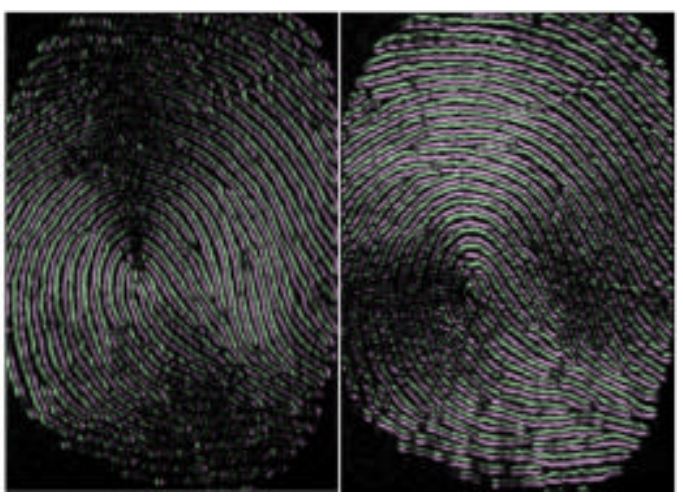

Fig. 8: The pictures for: a) The difference along $\mathrm{x}$ axis and b) The difference along $y$ axis, based on the image $500 \mathrm{dpi}$

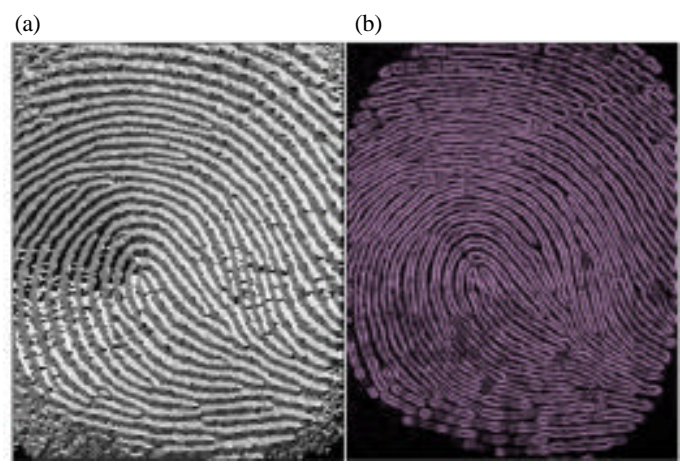

Fig. 9: a) Arguments of gradient ${ }^{(}(x, y)$ and $\left.b\right)$ Magnitude of gradient $m(x, y)$

$$
\mathrm{dy}(\mathrm{x}, \mathrm{y})=\mathrm{Sh}+\mathrm{Sb}-\mathrm{Sf}-\mathrm{Sd}
$$

The results $\mathrm{dx}$, dy and s are stored in new three layers using 500 dpi in the form of:

$$
\begin{aligned}
& \mathrm{dxt}\left(\frac{\mathrm{x}}{2}, \frac{\mathrm{y}}{2}\right)=\mathrm{dx}(\mathrm{x}, \mathrm{y}) \\
& \mathrm{dyt}\left(\frac{\mathrm{x}}{2}, \frac{\mathrm{y}}{2}\right)=\mathrm{dx}(\mathrm{x}, \mathrm{y}) \\
& \mathrm{S}^{\prime} \mathrm{t}\left(\frac{\mathrm{x}}{2}, \frac{\mathrm{y}}{2}\right)=\mathrm{S}^{\prime}(\mathrm{x}, \mathrm{y})
\end{aligned}
$$

Gradient calculation: In general, the gradient is used to describe the gray level variation in a fingerprint image. It can be applied to study the behavior of each block of pixels in terms of the value of its gradient in relation to flows of a fingerprint ridge. By Sulong and Saparudin (2015), the methods of gradient calculation are presented.

In this study, we calculate the gradient, using our results obtained in the previous paragraph. We used layers for $\mathrm{dx}$ and $\mathrm{dy}$ ( $500 \mathrm{dpi}$ ) to calculate the gradient direction through the following equations (Wang and Schroder, 2005):

$$
\begin{gathered}
\mathrm{m}(\mathrm{x}, \mathrm{y})=\sqrt{\mathrm{dx}_{\uparrow}^{2}(\mathrm{x}, \mathrm{y})+\mathrm{dy}_{\uparrow}^{2}(\mathrm{x}, \mathrm{y})} \\
\propto(\mathrm{x}, \mathrm{y})={ }_{0 \rightarrow 360}^{\operatorname{Arctg}}\left(\frac{\mathrm{dy}_{\uparrow}}{\mathrm{dx}_{\uparrow}}\right)
\end{gathered}
$$

This method has a number of advantages. First, there are no spaces between pixels when detecting a gradient; second, the calculation of differences at 1000 dpi makes it possible to build gradient at 500 more accurately. This will enhance the quality of a fingerprint image processing (Fig. 9).

\section{CONCLUSION}

An accurate estimation of the FOF is an essential step in the overall fingerprint recognition process. In this study, a new method to measure gradients was proposed. This method presents a different perspective on the problem of gradient estimation. The method is implemented in several stages to achieve the results. The first stage is the image enlargement to double resolution the second one is smoothing the image using the $5 \times 5$ filter; the third is calculating the $\mathrm{dx}$ and dy differences based on the $2 \times 2$ block, the fourth is up sampling and building the gradient. This method is realized in Visual Studio $\mathrm{C}++$. The results of image processing. Future work will focus on identifying loops and deltas based on the results of this study.

\section{REFERENCES}

Bian, W., S. Ding and Y. Xue, 2018. An improved fingerprint orientation field extraction method based on quality grading scheme. Intl. J. Mach. Learn. Cybern., 9: 1249-1260.

Can, X. and Y. Lin, 2009. An adaptive algorithm for smoothing fingerprint orientation fields. Proceedings of the 2009 International Conference on Computational Intelligence and Natural Computing, June 6-7, 2009, IEEE, Wuhan, China, ISBN:978-0-7695-3645-3, pp: 70-72. 
Cappelli, R., D. Maltoni and F. Turroni, 2010. Benchmarking local orientation extraction in fingerprint recognition. Proceedings of the 201020 th International Conference on Pattern Recognition, August 23-26, 2010, IEEE, Istanbul, Turkey, ISBN:978-1-4244-7542-1, pp: 1144-1147.

Cappelli, R., M. Ferrara and D. Maltoni, 2010. Minutia cylinder-code: A new representation and matching technique for fingerprint recognition. IEEE. Trans. Pattern Anal. Mach. Intell., 32: 2128-2141.

Doroz, R., K. Wrobel and P. Porwik, 2018. An accurate fingerprint reference point determination method based on curvature estimation of separated ridges. Intl. J. Appl. Math. Comput. Sci., 28: 209-225.

Gonzalez, R.C. and R.E. Woods, 2002a. Digital Image Processing. Pearson Education Inc., Upper Saddle River, NJ., USA.

Gonzalez, R.C. and R.E. Woods, 2002b. Digital Image Processing. 2nd Edn., Prentice Hall, Upper Saddle River, New Jersey, USA., ISBN:9780130946508, Pages: 793.

Gu, J. and J. Zhou, 2003. A novel model for orientation field of fingerprints. Proceedings of the 2003 IEEE Computer Society Conference on Computer Vision and Pattern Recognition Vol. 2, June 18-20, 2003, IEEE, Madison, Wisconsin, pp: II-493.

Hiew, B.Y., A.B.J. Teoh and O.S. Yin, 2010. A secure digital camera based fingerprint verification system. J. Visual Commun. Image Represent., 21: 219-231.

Imamverdiyev, Y., A.B.J. Teoh and J. Kim, 2013. Biometric cryptosystem based on discretized fingerprint texture descriptors. Expert Syst. Appl., 40: 1888-1901.

Jain, A.K., Y. Chen and M. Demirkus, 2007. Pores and ridges: High-resolution fingerprint matching using level 3 features. IEEE Trans. Pattern Analysis Mach. Intell., 29: 15-27.

Kayaoglu, M., B. Topcu and U. Uludag, 2015. Biometric matching and fusion system for fingerprints from non-distal phalanges. Comput. Vision Pattern Recognit., 2: 1-22.

Khfagy, M., Y. AbdelSatar, O. Reyad and N. Omran, 2016. An integrated smoothing method for fingerprint recognition enhancement. Proceedings of the International Conference on Advanced Intelligent Systems and Informatics, October 24-26, 2016, Springer, Cham, Switzerland, ISBN: 978-3-319-48307-8, pp: $407-416$.
Lee, S., H. Choi, K. Choi and J. Kim, 2008. Fingerprint-quality index using gradient components. IEEE. Trans. Inf. Forensics Secur., 3: 792-800.

Ma, J., X. Jun Jing, B. Zhang and S. Sun, 2010. An effective algorithm for fingerprint reference point detection. Proceedings of the 2010 2nd International Conference on Advanced Computer Control, March 27-29, 2010, IEEE, Shenyang, China, ISBN:978-1-4244-5845-5, pp: 200-203.

Maponi, P., R. Piergallini and F. Santarelli, 2017. Fingerprint orientation refinement through iterative smoothing. Signal Image Process. Intl. J., 8: 29-43.

Nagar, A., S. Rane and A. Vetro, 2010. Alignment and bit extraction for secure fingerprint biometrics. Proceedings of the International Conference on Media Forensics and Security II Vol. 7541, January 27, 2010 , SPIE, Bellingham, Washington, USA., pp: $1-14$.

Sulong, G. and Saparudin, 2015. Segmentation of fingerprint image based on gradient magnitude and coherence. Int1. J. Electr. Comput. Eng., 5: 1202-1215.

Tashk, A., M.S. Helfroush and M.J. Dehghani, 2010. A Chebyshev/Legendre polynomial interpolation approach for fingerprint orientation estimation smoothing and prediction. J. Zhejiang Univ. Sci. C., 11: 976-988.

Wang, Y., J. Hu and H. Schroder, 2005. A gradient based weighted averaging method for estimation of fingerprint orientation fields. Proceedings of the International Conference on Digital Image Computing: Techniques and Applications (DICTA'05), December 6-8, 2005, IEEE, Queensland, Australia, pp: 29-29.

$\mathrm{Xu}, \mathrm{H}$. and R.N.J. Veldhuis, 2010c. Binary representations of fingerprint spectral minutiae features. Proceedings of the 2010 20th International Conference on Pattern Recognition, August 23-26, 2010, IEEE, Istanbul, Turkey, ISBN:978-1-4244-7542-1, pp: 1212-1216.

$\mathrm{Xu}, \mathrm{H}$. and R.N.J. Veldhuis, 2010b. Binary spectral minutiae representation with multi-sample fusion for fingerprint recognition. Proceedings of the ACM 12th International Workshop on Multimedia and Security, September 09-10, 2010, ACM, New York, USA., ISBN: 978-1-4503-0286-9, pp: 73-80.

$\mathrm{Xu}, \mathrm{H}$. and R.N.J. Veldhuis, 2010a. Complex spectral minutiae representation for fingerprint recognition. Proceedings of the 2010 IEEE Computer Society Conference on Computer Vision and Pattern Recognition, June 13-18, 2010 , IEEE, San Francisco, California, USA., ISBN:978-1-4244-7029-7, pp: 1-8. 